\title{
1 Ziel und Anlage der Abhandlung
}

\subsection{Ziel und Spektrum der Abhandlung}

Texte über andere Texte (= Metatexte) haben grundsätzlich eine Doppelstruktur: Sie enthalten zum einen explizite Aussagen über andere Texte (= Phänotexte), zum anderen implizite Aussagen über den Blickwinkel und das Wertesystem des Verfassers des Metatextes. Betrachtet man mehrere Metatexte zu einem Phänotext, werden außerdem Grundzüge einer individuellen oder allgemeinen Rezeptionsgeschichte dieses Phänotextes erkennbar. Analysiert man eine Gruppe von Metatexten zu einer Gruppe von Phänotexten, darf man hoffen, zu allgemeineren Erkenntnissen über die Verfasser der Phäno- und Metatexte und deren Blickwinkel zu gelangen.

Diese Abhandlung versucht, aus exemplarischen japanischen literaturtheoretischen Texten der Jahre 1850 bis 1890 auf mögliche und gängige Blickwinkel sowie die Wertesysteme ihrer Verfasser zu schließen. Im Mittelpunkt soll dabei die Dichotomie „Fiktion - Wirklichkeit“ in ihren unterschiedlichen Formulierungen und den jeweils damit verbundenen Begriffsinhalten stehen. Mein Interesse an dieser Dichotomie rührt von ihrem grundsätzlichen Charakter und ihren damit verbundenen Fragestellungen her. Denn wenn ein literarischer Text grundsätzlich als Fiktion im Sinne eines Artefakts ( $\leftarrow$ lat. facere, „herstellen“) betrachtet werden darf, ergibt sich die Frage: Welche Beziehung konstruiert der Literaturtheoretiker zwischen dem „Hergestellten“ und dem außerhalb des Textes „schon Seienden“, also der Wirklichkeit? Oder, noch pathetischer formuliert: Welchen Bezug zwischen dem literarischen Text und der „Welt“ konstruiert der Literaturtheoretiker? Im oben grob umrissenen Zeitraum der Jahre 1850 bis 1890 unterliegt die japanische „Welt“ auf innen- und außenpolitischer, geistesgeschichtlicher und kultureller Ebene drastischen Veränderungen. Ziel der vorliegenden Abhandlung ist die Erkenntnis der damit einhergehenden Veränderungen der Begriffsinhalte der Dichotomie „Fiktion - Wirklichkeit“ in der japanischen Literaturtheorie der Zeit. Geht es in den zu betrachtenden japanischen Literaturtheorien um derart Grundsätzliches, dann übernimmt die vorliegende Abhandlung die von Ulrike Zeuch in Bezug auf die Rezeption der Poetik des Aristoteles und speziell auf dessen Mimesis-Begriff bezogen entwickelte Vorstellung von einer „Tauglichkeit literaturtheoretischer Begriffe zur Beschreibung ideengeschichtlicher Prozesse“. Eine japanische Form der Mimesis etwa stellte sich damit als kulturanthropologische Konstante dar, deren Literaturtheorie gar als kulturanthropologische Literaturtheorie. ${ }^{1}$ Es muss dabei

1 ZeUCH 2010. Auf diesen Gedanken komme ich in der Mitte (in Kapitel 4) und am Ende der Abhandlung zurück.

Ә Open Access. (C) 2022 Guido Woldering, publiziert von De Gruyter. (c) BY-NC-ND Dieses Werk ist lizenziert unter einer Creative Commons Namensnennung - Nicht-kommerziell - Keine Bearbeitung 4.0 International Lizenz. https://doi.org/10.1515/9783110763942-002 
klar sein, dass die vorliegende Abhandlung eine Übersicht thematischer Schwerpunkte der auf die Dichotomie „Fiktion - Wirklichkeit“ bezogenen Literaturtheorien und den daraus möglichen Folgerungen bieten wird - nicht mehr und nicht weniger.

Die folgenden (von mir übersetzten) Ausschnitte japanischer Metatexte der Jahre 1850 bis 1890 zur japanischen Literatur (im Sinne von Wortkunst) dienen als erstes Schlaglicht auf drei wichtige Blickwinkel und Wertesysteme ihrer Verfasser in drei voneinander zu unterscheidenden Gruppen A, B und C. Auch erlauben sie eine erste Abgrenzung zwischen nichttheoretischen und theoretischen Metatexten zur Literatur.

Tabelle 2: Sechs einführende Beispiele für Metatexte zur japanischen Literatur (1849-1889).

1

A Nun, was die historisierenden Erzählungen anbetrifft, so schaffen sie vermittels einer Entleihung das Wahre. Was [ihre] Metaphern, ihre rätselhaften und vergleichenden Ausdrücke, das in der Luft Befestigte und das Wurzellose angeht, so scheint daran nichts Beachtenswertes zu sein, doch veranschaulichen die feinen Formulierungen die Beförderung [des Guten] und die Geißelung [des Schlechten] und gereichen den Unterbelichteten und Irregeleiteten zur Unterweisung. [...] Dies war der Ursprung der Heftliteratur. (Ishikawa Teisai: Vorwort zu Kimura Mokurō, Kokuji shōsetsu tsū, 1849)

B Will man diese Erzählung [das Genji monogatari] lesen, so sollte man sich zuerst die historische Situation gründlich einprägen und sie begreifen und erst dann mit der Lektüre beginnen. Geschieht dies nicht, so weichen die [geschilderten] Verhältnisse erheblich [von der historischen Situation] ab, es kommt zu vielen Verwirrungen in den Herzen späterer Generationen, und man kann den Sinn schwerlich erfassen. [...] [Aber] weil alles so niedergeschrieben wurde, wie es sich in alten Zeiten zugetragen hatte, fühlt man sich in jene Zeit zurückversetzt. So wirkt es, und deswegen gibt es vieles, dessen Sinn man als das erfasst, was die Autorin zu ihrer Zeit erlebt hatte. (Hagiwara Hiromichi: Genji monogatari hyōshaku, 1854)

\section{2}

Fasst man das Hauptanliegen [meines Romans] zusammen, so habe ich nicht im Traum daran gedacht, leere Worte, Gelogenes und Spielereien hervorzubringen - sondern nur daran, [für die ungebildete Leserschaft] möglichst kurze und einfache Beförderungen des Guten und Geißelungen des Bösen zu schaffen. (Shōtei Kinsui: Temari uta sannin musume, Vorwort zu Bd. II [postum, 1864])

Neulich besuchte ich Herrn Professor Keiu und befragte inn zu Poesie und Prosa. Der Herr Professor merkte an, dass er an Poesie und Prosa das Tatsächliche schätze, die Vorstellung hingegen nicht. Ich sage bei aller gebotenen Bescheidenheit, dass ich bei Poesie das Vorgestellte, nicht aber das Tatsächliche schätze. Das Tatsächliche ist nur in der Prosa zu schätzen. [...] Das ist der Grund, warum der Poet in westlichen Ländern die Vorstellung zur Hauptsache macht. (Inoue Tetsujirō, Nakamura Keiu ō ni yosete sho su, 1883) 
Tabelle 2 (fortgesetzt)

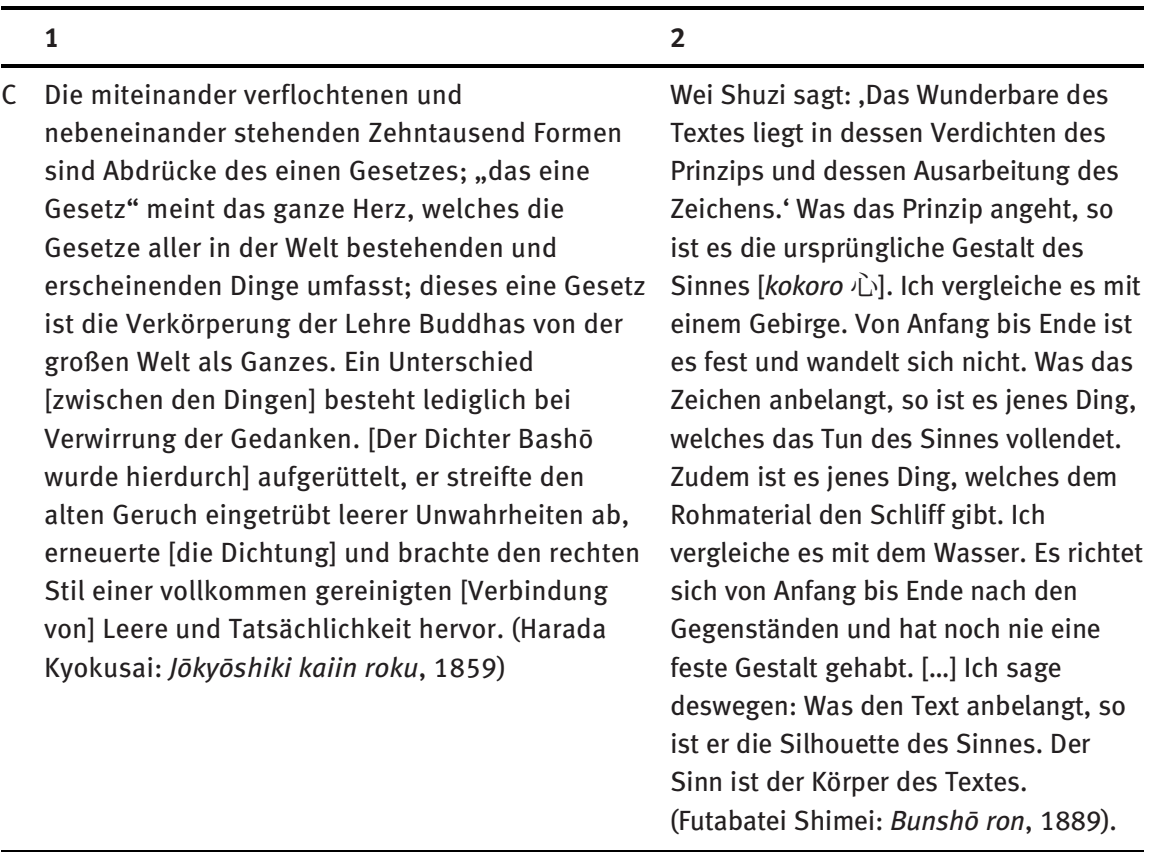

\section{Gemeinsamkeiten der sechs einführenden Metatexte}

Betrachten wir zunächst die Gemeinsamkeiten der sechs Texte! Alle sprechen über die Wortkunst (= Literatur), und alle thematisieren das für die Literatur konstatierte oder geforderte Verhältnis zwischen Fiktion und extratextueller Wirklichkeit, zwischen Fiktivität und Faktizität. Gemeinsam ist den Texten zudem die Entstehungszeit, nämlich die im Mittelpunkt der Abhandlung stehenden Jahre 1850-1890, eine Zeit des wesentlich von ausländischen Einflüssen geprägten, politisch und sozialgeschichtlich eher abrupten, literaturgeschichtlich eher allmählichen Übergangs von der späten Feudalzeit zur frühen Moderne Japans. Vergleicht man nun gruppenweise die älteren Texte der Spalte 1 mit den neueren der Spalte 2, stellt man Übereinstimmungen in den zugrundeliegenden Wertesystemen fest. (A) Der ganz und gar der traditionellen Feudalmoral verhaftete Ishikawa Teisai 石川艇斎 (fl. 1849) lässt 1849 Fiktion in der Literatur als „Entleihung“ der extratextuellen Wirklichkeit nur so weit zu, wie sie den Ungebildeten zur leichtverständlichen moralischen Unterweisung dient - ein Standpunkt, den Shōtei Kinsui 松亭金水 (1797-1863) mitten in der Übergangsphase in seinem postum veröffentlichten Text noch vollkommen zu teilen vorgibt, um die Zensurbehörden und die 
Leserschaft für seine Produkte einzunehmen. (B) Der 1854 noch tief in der Feudalzeit verwurzelte Hagiwara Hiromichi 萩原広道 (1815-1863) setzt die enge Beziehung des fiktionalen Genji monogatari zur gesellschaftlichen Realität des höfischen Lebens im 10. Jahrhundert als gleichsam naturgegeben voraus - worin 1883 Nakamura Keiu 中村敬宇 (1832-1891), prominenter Vertreter der Aufklärung der Frühmoderne (Meiji-Zeit, 1868-1912) mit ihm noch völlig übereinstimmt, sein Diskussionspartner Inoue Tetsujirō 井上哲次郎 (1856-1944) als ein Vertreter der frühmodernen Philosophie Japans zumindest noch großenteils. (C) Futabatei Shimei 二葉亭四迷 (1864-1909) sagt im Jahre 1889, also in der Frühmoderne eigentlich noch dasselbe wie schon Harada Kyokusai 原田曲斎 (1817-1874) gegen Ende der Vormoderne Japans: dass das extratextuelle Referens des literarischen Texts vor allem der dem referierten Gegenständlichen innewohnende Sinn sei und nicht das Gegenständliche selbst. Die jeweils Jahrzehnte auseinanderliegenden Texte einer Gruppe verweisen auf drei vorherrschende Literaturanschauungen des Betrachtungszeitraums: (A) eine moralisch, (B) eine allgemein geschmacklich und (C) auf eine im engeren Sinne ästhetizistisch oder philosophisch urteilende Anschauung. Dass diese drei Anschauungen ihren Ursprung in weit früheren historischen Phasen haben und sich im konkreten Fall vermischen können, sei zunächst ohne Beleg erwähnt.

Eine weitere wichtige Gemeinsamkeit der sechs Beispieltexte: Die Lebenszeiten der Verfasser überlappen sich zu einem großen Teil, und ihre Texte verweisen - bei aller sozial und biografisch bedingt individuellen Ausprägung - auf Gemeinsamkeiten in Bildungsweg und Sozialisierung. Vor diesem Hintergrund überraschen mehr noch als deren Gemeinsamkeiten die Unterschiede zwischen den Metatexten.

Die dritte Gemeinsamkeit der Texte der Gruppen A bis C ist der Bezug zur „Literatur“ im Sinne von „Wortkunst“. Bevor wir uns in diesem womöglich unendlich scheinenden Feld verlieren, muss dessen Definition nun nachgeholt werden. Der Umgang mit den verschiedenen Formen der japanischen Wortkunst legt nahe, sich an Wolfgang Isers im Folgenden zitierter Erklärung aus Die Appellstruktur der Texte (1970) zu orientieren.

[Literarische Texte] besitzen keine genaue Gegenstandsentsprechung in der ,Lebenswelt", sondern bringen ihre Gegenstände aus den in der ,Lebenswelt‘ vorfindbaren Elementen erst hervor. [...] es gibt durchaus Texte, die etwas hervorbringen, ohne dadurch schon literarisch zu sein. So schaffen beispielsweise alle Texte, die Forderungen stellen, Ziele angeben oder Zwecke formulieren, ebenfalls neue Gegenstände, die jedoch erst durch das vom Text entwickelte Maß an Bestimmtheit ihren Gegenstandscharakter gewinnen. Gesetzestexte bilden den paradigmatischen Fall solcher Formen der Sprachlichkeit. Das von ihnen Gemeinte gibt es dann als verbindliche Verhaltensnorm im menschlichen Umgang. Im Gegensatz dazu vermag ein literarischer Text niemals solche Sachverhalte zu schaffen. 
Kein Wunder also, daß man diese Texte als Fiktionen bezeichnet, denn Fiktion ist Form ohne Realität. [...] Seine [des literarischen Texts] Realität gründet nicht darin, vorhandene Wirklichkeit abzubilden, sondern darin, Einsichten in diese parat zu halten. [...] [Der literarische Text] unterscheidet sich einerseits von anderen Textarten dadurch, daß er weder bestimmte reale Gegenstände expliziert noch solche hervorbringt, und er unterscheidet sich andererseits von den realen Erfahrungen des Lesers dadurch, daß er Einstellungen anbietet und Perspektiven eröffnet, in denen eine durch Erfahrung gekannte Welt anders erscheint. So läßt sich der literarische Text weder mit den realen Gegenständen der ,Lebenswelt' noch mit den Erfahrungen des Lesers vollkommen verrechnen. Die mangelnde Deckung erzeugt ein gewisses Maß an Unbestimmtheit. Diese allerdings wird der Leser im Akt der Lektüre ,normalisieren'. ${ }^{2}$

Damit wird aus der Betrachtung das ursprünglich gleichfalls zur „Literatur“ gehörende Feld der wissenschaftlichen Texte ausgeschlossen. Das ist auf den ersten Blick zweifellos problematisch, denn dass es sowohl im Deutschen als auch im Japanischen je einen die beiden Teilfelder umschließenden, historisch gewachsenen Oberbegriff gibt (im Japanischen ist es bungaku 文学), verweist ja auf eine wenigstens partielle Übereinstimmung der Teilfelder. Angesichts dieser partiellen Übereinstimmung ist es erstaunlich, dass der Kontrast ausgerechnet der beiden offensichtlich doch verwandten Teilfelder immer wieder herangezogen wird, um das jeweils andere Teilfeld zu charakterisieren. Und dabei gelingt die Abgrenzung auch noch selten vollständig. ${ }^{3}$ Dies sollte im Blick behalten werden, wenn im Zusatz zu Isers Charakterisierung vereinfachend als wichtigste Merkmale der Literatur im Sinne von Wortkunst deren ausgeprägte Autoreferentialität, der als ästhetisches Verfahren gezielt eingesetzte Isotopiebruch und die

2 ISER 1974 [1970]: 10-12.

3 Beispielsweise verzichtet René Wellek nur der klaren Abgrenzung von Wissenschaft und Literatur zuliebe darauf, ausführlicher auf die Überlappung der beiden Felder einzugehen. Den wichtigsten Unterschied sieht er bei der jeweiligen Beziehung zwischen Zeichen und Bezeichnetem: Wissenschaft strebe eine Übereinstimmung der beiden an, Literatur dagegen verweise auf das Zeichen selbst (WELLEK-AUSTIN 1985: 17-27). Hinzu kommt für Wellek noch ein Unterschied in der (vor allem von Vertretern anderer Wertgebiete aufgeworfenen) Frage der Funktion von Literatur: Im Gegensatz zur Wissenschaft habe sie nach Meinung der Apologeten der Literatur zusätzlich zum Nutzen der Belehrung auch den Nutzen des Erfreuens (WELLEK-AUSTIN 1985: 27-38). Dass diese Merkmale auch in nichtliterarischen Texten zu finden sind, wird zwar nicht bestritten, aber auch nicht weiter diskutiert. Welleks Thesen erscheinen nur vorübergehend als terminologisches Instrumentarium einsetzbar und müssen im Verlauf der Arbeit ergänzt werden. Das gilt auch für Jürgen Links Abgrenzung der Literatur von der wissenschaftlichen Rede. Von letzterer fordert Link (1) die Definition aller Klasseme im Text (also die Identität von Klassemen und Aktanten), (2) eine in sich widerspruchsfreie Fügung der Klasseme und (3) die Möglichkeit, die Struktur der Klasseme zu einem Instrumentarium empirischer Beobachtungen zu machen (LinK 1985: 82). 
große Distanz zwischen Bezeichnung (Signifikant) und Bezeichnetem (Signifikat) gelten sollen. Die durch die obigen Gruppen A bis $\mathrm{C}$ vertretenen und für die vorliegende Abhandlung ausgewählten Metatexte haben gemeinsam, auf eben die soeben genannten Merkmale der Wortkunst zu reagieren, sich also mit solchen Texten zu befassen, die von der extratextuellen Welt deutlich abzugrenzen sind und durch ästhetische Verfahren eine große Distanz zwischen Signifikant und Signifikat gezielt erzeugen.

Damit soll der begriffliche Inhalt des deutschen Wortes „Literatur“ als für die Abhandlung operationalisierbar gelten. Zur Absicherung gegen die Gefahr, die japanische Literatur in ein terminologisches Prokustesbett zu zwingen, wäre eine japanische Entsprechung des Begriffes „Literatur“ wünschenswert. Das erwähnte Wort bungaku leistet diese Absicherung aber nur bedingt, gewissermaßen als Orientierungshilfe, denn zwar wird es in Bezug auf die japanische Moderne meist im oben gegebenen Sinne von „Wortkunst“ verwendet, darin dem deutschen Wort „Literatur“ entsprechend. ${ }^{4}$ Doch erscheint bungaku als Terminus nur auf den ersten Blick wie eine Entsprechung zu „Literatur“. Denn die mit der wörtlichen Bedeutung („Schriftgelehrsamkeit“) einhergehende begriffliche Breite schließt lange Zeit die Teilfelder „Wissenschaft“ und nur einen Teil der „Wortkunst“ ein. ${ }^{5}$ Darin, dass bungaku von Anfang an nicht alle Formen der Wortkunst einschloss und insbesondere die fiktionale Prosa ausschloss, liegt der wesentliche Unterschied zum Wort „Literatur“. Auch ist die Begriffsgeschichte des Wortes bungaku wechselvoll und führt nicht auf geradem Wege zur Eingrenzung auf das Teilfeld „Wortkunst“. Der erste belegte Gebrauch des Wortes verweist in der Gesetzessammlung Yōrō ritsuryō 養老律令 (720) auf den Schriftgelehrten, der die Prinzen und Prinzessinnen in den Kanon der klassischen chinesischen Literatur einwies. ${ }^{6}$ Als Oberbegriff für das chinesische und damit im sozialen Prestige gehobene wissenschaftliche und nichtwissenschaftliche Schrifttum wird bun in bungaku erstmals im Vorwort der kanshi-Sammlung Kaifūsō 懐風藻 (Fond Recollections of Poetry, 751) verwen-

\footnotetext{
4 Diese Zuordnung beginnt in Japan erst mit der Einführung westeuropäischer Wissenschaftsbegriffe durch Nishi Amane 西周 (1829-1897) in seiner Vorlesung Hyakugaku renkan 百学連環 ([Die hundert Verbindungsringe der Gelehrsamkeiten], 1870). (FujIHIRA 1965: 14).

5 Das Bewusstsein für die ursprüngliche begriffliche Breite ist nicht verschwunden, wie Suzuki Sadami anhand moderner japanischer Standardnachschlagewerke belegen kann (SUZUKITYLER 2006: 11-18).

6 NST 3: 207-208. Fumihakase hitori. Tsukasadoramu koto, kyō wo torite, kōju semu koto. 文学一 人。掌。執経講授。 „Ein Lettré / Er hat die Aufgabe, an Hand der klassischen Schriften [die kaiserlichen Prinzen und Prinzessinnen] interpretierend zu belehren.“ (DETTMER 2009-I: 365).
} 
det, verweist dort aber ausschließlich auf Lyrik. ${ }^{7}$ Nichtwissenschaftliche chinesische Prosa dagegen wird im 8. Jahrhundert häufig bunshō 文章 (chin. wenzhang) genannt. Im Grunde setzt sich die Tradition, mit bungaku das in gehobener (also chinesischer) Sprache verfasste Schrifttum zu bezeichnen, bis zum Anfang des 17. Jahrhunderts fort. ${ }^{8}$ Das von portugiesischen Missionaren geschaffene Vocabulario da Lingoa de Iapam com Adeclaração em Portugues 1603; in der faksimilierten Edition im Haupttitel Nippo jisho 日葡辞書 ([Japanisch-Portugiesisches Lexikon]), reflektiert das zeitgenössische japanische Verständnis und erläutert bungacu [= bungaku] über die Phrase fumi manabu [文学] als Bezeichnung für Studien, Schriftgelehrsamkeit und das Kultivieren eines schönen Stils (Estudo sciencia de livros - bom estillo de cartas), doch die „stilistische Schönheit“ des gehobenen Chinesischen entsprach dabei der „Schönheit“ des gehobenen Lateinischen im Kontrast zur vulgären portugiesischen Alltagssprache. ${ }^{9}$ In der EdoZeit (1603-1867) verengt sich der Begriffsgehalt von bungaku wieder auf konfuzianische Schriftgelehrsamkeit, während für Lyrik und Prosa in chinesischem Stil (kanshi 漢詩 und kanbun 漢文) Bezeichnungen wie geibun 芸文 (,künstlerische Texte“) und bungei 文芸 (,Textkunst“) häufiger Verwendung finden. ${ }^{10}$

Die wechselvolle Begriffsgeschichte legt es nahe, das Wort bungaku in der vorliegenden Abhandlung je nach Kontext mit „Literatur“ im Sinne von „Wortkunst“ oder mit „Schriftgelehrsamkeit“ zu übersetzen und den Unterschied bei der Zusammenstellung des Untersuchungskorpus’ zu berücksichtigen.

7 Der in unserem Zusammenhang zentralen Passage geht eine Darlegung der durch Kaiser Tenji tennō 天智天皇, (Ōmi 青海 626-672; reg. 661-672) eingeleiteten Reformen voraus. Dieser habe der Entwicklung der säkularen Kultur einen unter der Vorherrschaft des Buddhismus bislang unmöglichem Maße Raum gegeben. Originaltext: Koko ni sankai heikan, shikai inshō, ryūkō mui, ganrai itoma ōshi. Shibashiba bungaku no onoko wo oki, tokidoki chirei no asobi wo hirakitamau. Kono kiwa ni atarite, shinkanbun wo tarashi, kenshin shō wo tatematsuru. (NKBT 69: 59-60). „Then the Three Classes enjoyed peace and glory; within the Four Seas reigned prospertiy and wealth. The great dignitaries had surcease[d] from their prosperity and wealth. The palace galleries knew much leisure. At times the emperor summoned men of letters; often great banquets were held. On these occasions the imperial brush let fall prose; the courtiers offered their eulogies in verse“ (TSUNODA-DE BARY-KEENE 1958-I: 89). Aus dem Zusammenhang ergibt sich, dass bungaku zwar auch hier Personen (men of letters) bezeichnet, der Bestandteil bun aber nicht, wie das soeben erwähnte Ritsuryō, die wissenschaftliche Schriftgelehrsamkeit.

8 SUZUKI-TYLER 2006: 57-68.

9 Suzuki Sadami gibt nur einen Teil der hier zitierten Definitionen wieder (SUZUKI-TyLER 2006: 68). Quelle des Originals: KAMEI 1975 [1603]: s.v. bungacu.

10 Dies ist etwa in den Schriften des konfuzianischen Gelehrten Emura Hokkai 江村北海 (1713-1788) der Fall (SUZUKI-TYLER 2006: 76). 


\section{Unterschiede zwischen den Textbeispielen - Anschauung vs. Theorie}

Nachdem wir die wichtigsten Gemeinsamkeiten der in den Gruppen A, B und C gegebenen Textausschnitte in den Blick genommen haben, sollen nun deren Unterschiede zur Sprache kommen. Dafür sind zunächst die „Anschauung“ und die wissenschaftliche „Theorie“ voneinander $\mathrm{zu}$ trennen, gerade weil das griechische Wort theoría dem deutschen „Anschauung“ etymologisch nahekommt. Als Theorie soll künftig ein Metatext gelten, der seine Anschauung nicht aus der Empirie, sondern aus dem Denken über das Beobachtete gewinnt. Eine Theorie teilt die Welt in Beobachtung und (allerdings davon abhängiges) Beobachtetes auf ${ }^{11}$ und formuliert Gesetzmäßigkeiten, die eine bestimmte Klasse beobachteter Erscheinungen hervorbringen. Je nachdem, wie viele Einzelgesetze eine Theorie in sich zusammenfasst, vergrößert sich die Klasse der von ihr behandelten Erscheinungen jedenfalls aber unterscheidet ihr Anspruch auf eine über den betrachteten Einzelfall hinausgehende Gültigkeit die Theorie von der Anschauung. Während übrigens letztere das Vorhandensein einer richtigen Antwort impliziert, stellt die Theorie einen argumentierenden Erklärungsversuch dar, der in einem hermeneutischen Zirkel Gegenstand der nächsten Beobachtungsstufe werden kann. Gegenstand der Theorie ist demnach überwiegend nicht das Phänomen an sich, sondern die Form, in der es wissenschaftlicher Gegenstand wird. ${ }^{12}$

Die oben gegebenen Textausschnitte der Gruppen A bis C zitieren jeweils „nur“ die Kernaussage des Gesamttexts, doch sind Blickwinkel sowie Wertesysteme ihrer Verfasser erkennbar, und ihre Texte können grob in Anschauung und Theorie geschieden werden. (A) Sowohl in Ishikawa Teisais Vorwort zum Kokuji shōsetsu tsū (Kimura Mokurō, 1849) als auch in Shōtei Kinsuis Vorwort $\mathrm{zu}$ Bd. II seines Temari uta sannin musume ([1864]) ist die fiktionale Prosa mit moralischer Wirkung verbunden, aber Kinsui erklärt nicht, wie „Gelogenes und Spielerei“ im Temari uta mit dieser Wirkung verbunden sind, während Teisai durch die Nennung der Metaphorik ein formales Kriterium (das tertium comparationis zwischen Fiktion und Wirklichkeit) wenigstens andeutet. Dies macht Teisais Text zu einem (wenn auch schwach fundierten) literaturtheoretischen Text, Kinsuis Text verlässt nicht das Gebiet der Anschauung. (B) Hagiwara Hiromichi will 1854 noch immer die höfische Erzählung Genji monogatari (ca. 1010) als Abbildung der Lebenswirklichkeit ihrer Autorin verstanden wissen, erklärt aber nicht, warum der schon im Titel als „in Form gebrachte Fiktion“ (monogatari, worin gatari sich aus kata ari oder „hat eine Form“ herleitet) gekennzeichnete

11 JAHRAUS 2004: 64.

12 Jahraus 2004: 65. 
Text sich hierin von Historien (shi 史) nicht unterscheiden soll. Diese Anschauung der Prosa als Medium des Sachtextes übernimmt Inoue 1883 zwar noch, gesteht aber dafür zumindest der Lyrik („Poesie“) die Fiktionalität (,das Vorgestellte“) zu. Das Gebiet der Anschauung verlassen jedoch beide Texte nicht, denn in Inoues Text fehlen sowohl die Konstruktion des Zusammenhangs zwischen Fiktion und Wirklichkeit als auch jegliche Argumentation, in Hiromichis Text fehlt zumindest eine Argumentation. (C) Sowohl Kyokusais Jōkyōshiki kaiin roku (1859) als auch Futabateis Bunshō ron (1889) sind im definierten Rahmen als Literaturtheorien zu betrachten. Beide trennen Signifikant und Signifikat des literarischen Textes voneinander und argumentieren ihre Sicht auf den jeweiligen substantiellen Kern der beiden Teile sowie auf die Beziehung der Kerne zueinander. Kyokusai zitiert aus dem Buddhismus das eine, allumfassende und unwandelbare Gesetz (ippō 一法), das es im Gedicht zu versprachlichen gelte; Futabatei nennt in ähnlicher Weise die „Verdichtung des Prinzips“ (sekiri 積理) als Aufgabe des Textes allgemein (bunshō 文章), wobei $r i$ hier noch der nekonfuzianische Begriff des allem Sein zugrundeliegenden Prinzips ist.

Ein caveat ist nach diesen terminologischen Eingrenzungen allerdings doch nötig. Für den Fall der japanischen Literaturtheorie des Zeitraums 1850-1890 soll festgehalten werden, dass die im Mittelpunkt stehenden Gesetzmäßigkeiten in der Theorie nicht notwendigerweise in einer nach westlichem Verständnis expliziten und eindeutigen Weise formuliert sein müssen, sondern es im Extremfall ausreichen kann und muss, wenn sie sich zwingend logisch ergeben. ${ }^{13}$ Analog gilt dies auch für die sprachliche Gestalt der Literaturtheorie: Schon eine oberflächliche Sichtung in Frage kommender Texte offenbart eine große Vielfalt, zu der abstrakte Darlegungen in wissenschaftlich-trockener Prosa (oben Text A-Ishikawa 1849) ebenso gehören wie metaphorisch und gar in gebundener Sprache (oben Texte C) formulierte Literaturtheorien. Ein Ausschluss der letztgenannten Gruppe würde nach meiner Überzeugung zu einem unvollständigen Bild nicht nur der

13 Damit wende ich einige Merkmale der japanischen Syntax analog auf die Literaturtheorie an. So sind in der japanischen Regelsyntax Bezeichnungen von Subjekt und Objekt fakultativ, Rückverweise auf bereits eingeführte Begriffe geschehen häufig implizit, und selbst formalgrammatisch erwartbare Finalformen können entfallen, weswegen Ellipsen im Japanischen keine Seltenheit sind. All dies wird in der Regel von der Sprechergemeinschaft nicht als Unbestimmtheit empfunden, weil der Einbezug von Ko- und Kontext seitens des Rezipienten vorausgesetzt wird. Prinzipiell gilt das selbe für Quellenverweise: Den Verweis auf Autor, Titel und Erscheinungsort spart sich der Verfasser häufig, weil er von einem (dann allerdings nicht belegten) homogenen Wissensstand seiner Leser ausgeht. Form, Inhalt und Funktionieren von Quellenverweisen in der vormodernen japanischen Literatur scheinen mir genau wegen der implizierten Homogenität der Leserschicht ein Desideratum der Literaturforschung zu sein. 
Literaturtheorie, sondern des Denkens in Japan überhaupt führen. Auch müssen wir akzeptieren, dass das theoretische Niveau literaturtheoretischer Texte unterschiedlich sein kann, soweit sich dies etwa in der argumentativen Konsistenz manifestiert.

\subsection{Der historische Rahmen der Abhandlung}

Dass die Betrachtung von Konstanten und Wandlungen in Umbruchzeiten besondere Aufschlüsse versprechen, liegt auf der Hand, denn Konstanten lassen auf Grundwerte, Wandlungen auf Entwicklungspotenzial schließen. Aber dass ich ausgerechnet Literaturtheorien der Zeit des Wandels der Jahre 1850 bis etwa 1890 als Forschungsgegenstand wähle, bedarf doch einer Erklärung. Denn angefangen mit den Poetiken des 10. Jahrhunderts gibt es in Japan eine Literaturtheorie ja beinahe so lange wie ihren Gegenstand selbst. ${ }^{14}$ Aber sowohl Anfang wie Ende des Betrachtungszeitraums werden durch deutliche Einschnitte in die gesellschaftliche und kulturelle Geschichte Japans markiert, die es rechtfertigen, die dazwischenliegende Zeit als eine besonders reizvolle historische Übergangsphase zu behandeln.

Die gesellschaftliche Situation Japans ist zur Mitte des 19. Jahrhunderts geprägt von gescheiterten Wirtschaftsreformen, Hungersnöten, Bauernaufständen und die nach dem britisch-chinesischen Opium-Krieg offenkundige Bedrohung der staatlichen Souveränität Japans durch westliche imperialistische Staaten, ${ }^{15}$ die ab den Fünfziger Jahren des 19. Jahrhunderts die Abschottungspolitik Japans untergruben und ihr schließlich ein Ende setzten. ${ }^{16}$ Auch die Literaturgeschichte erfährt in dieser Zeit eine deutliche Zäsur. Die kommerzialisierte unterhaltende Erzählprosa - in einer captatio benevolentiae von den Autoren als „nur zum Spaße geschrieben“ (gesaku 戯作) bezeichnet - ist in Produktionsmenge und Verbreitung marktbeherrschend, ${ }^{17}$ als im Jahr 1848 mit Takizawa Bakin 滝沢馬琴 (geb. 1767) der Nestor gehobener Erzählprosa und insbesondere des yomihon (eines an chinesische Vorbilder angelehnten, sprachlich äußerst anspruchsvollen

14 Siehe auch BENL 1951 und UEDA 1991. UEDA 1991 beschränkt sich zwar im Gegensatz zu BENL 1951 nicht auf die Literaturtheorie der Lyrik (also auf die Poetik), behandelt aber wie jener keine Literaturtheorien der japanischen Frühmoderne.

15 BOLITHO 1989.

16 BeAsley 1989.

17 MAY 1983. 
Abenteuerromans) stirbt. ${ }^{18}$ Dessen bekanntestes yomihon ist das Nansō Satomi hakkenden 南総里見八犬伝 (Eight Dogs, 1814-1842), ein immens umfangreicher Long- und Bestseller, in dem Bakin Elemente aus chinesischen Räubererzählungen zu einem phantastischen Roman vor der historischen Kulisse kriegerischer Auseinandersetzungen im Japan des 15. Jahrhunderts verarbeitet. In den Begleittexten (Vorworten, intermittierenden Kommentaren und Nachworten) legt Bakin, teilweise auf gerade erhaltene Zuschriften eingehend, historische Fakten, ethischmoralische Leitlinien und literaturtheoretische Überlegungen als Grundlagen seiner Textgestaltung einzelner Kapitel oder größerer Erzählstränge dar. ${ }^{19}$ Der letzte Begleittext dieser Art ist der auf das Gesamtwerk bezogene und es abrundende Abschnitt Kaigai jōhitsu 回外剰筆 ([Außerhalb der Kapitel zusätzlich Geschriebenes]; Sammlung 9, Band 53), den der inzwischen erblindete Bakin seiner Schwiegertochter diktiert. Neben zahlreichen historischen und philologischen Schlussbemerkungen fasste Bakin hier in Form der Traumerzählung eines ihn besuchenden Mönchs seine Vorstellungen von Fiktion und Wirklichkeit zusammen, um Abweichungen eines literarischen Textes von historischen Fakten zu legitimieren: Es ist die überraschend stereotype defensive Argumentation, die wir schon in Gruppe A der Beispieltexte kennengelernt haben - und die im Prinzip schon im 10. Jahrhundert einen festen Platz im Kanon der Literaturtheorie hatte. Am Ende der Hochblüte des yomihon übernimmt das Kaigai jōhitsu die Rolle eines literaturtheoretischen Abgesangs auf traditionelle Vorstellungen von Erzählprosa. Denn was während der sich daran anschließenden letzten zwei Dekaden der Feudalzeit (künftig EdoZeit, 1850-1868) literarisch und literaturtheoretisch produziert wurde, war epigonal und nicht geeignet, auf literarischem und literaturtheoretischem Gebiet mit dem Tempo der radikalen gesellschaftlichen Umwälzungen mitzuhalten, welche die Öffnung des Landes gegenüber westlichen Großmächten und schließlich das Ende der Feudalherrschaft herbeiführten. ${ }^{20}$

$18 \mathrm{Zu}$ Bakin sind überaus viele Monografien und Beiträge erschienen, die weder hier noch im Literaturverzeichnis erschöpfend aufgeführt werden können. Als Höhepunkt der aktuellen japanischen Bakin-Forschung ist ITASAKA 2010 zu sehen.

19 Ein Beispiel sind die „Sieben Prinzipien des historisierenden Romanes“ (haishi shichi hōsoku 稗史七法則), die allerdings weder als synchron funktionierendes System, noch als Literaturtheorie im engeren Sinne gelten können, sondern vielmehr als Erläuterungen des „Handwerks“ der Leserbindung (WoLDERING 1993). Selbst das siebente Prinzip, welches im Gegensatz zu den anderen nicht den Techniken der Protagonistenkonstellation, der Motivverknüpfung und der Vorausdeutung gewidmet ist, sondern dem nicht Gesagten und dem Unsagbaren, erreicht wie auch die übrigen nicht das eingangs definierte Gebiet der Literaturtheorie.

20 Ausführlich hierzu OKITSU 1960 und OKITSU 1976. Warum die auf diese Weise charakterisierte Verfallsepoche aus literaturhistorischer Sicht um nichts weniger betrachtenswert ist, sollte sich aus den Ergebnissen der vorliegenden Arbeit erhellen - es kann in der Forschung 
Das Ende der für diese Abhandlung gewählten Betrachtungszeit ist das Jahr 1890. Politisch markiert es den Abschluss der Transformation Japans von einem aus über dreihundert mehr oder weniger selbstständigen Fürstentümern gebildeten Feudalstaat ${ }^{21}$ in einen modernen, zentralistisch geführten, industriekapitalistischen und in der Folge imperialistischen Staat. ${ }^{22}$ Die feudale Ständeordnung ist nun größtenteils beseitigt, unter einer oligarchischen Staatsführung entstehen große Industriekonzerne, ein in Ballungsgebieten konzentriertes Industrieproletariat und gleichzeitig auch erste politische Parteien. Kulturgeschichtlich kommt um 1890 eine Phase der stark an Europa und den USA orientierten Modernisierung ${ }^{23}$ zum Abschluss, und Japan beginnt nun mit der Suche nach einer neuen, eigenen Identität.

Nach dem hiermit umrissenen Weg vom Verfall traditioneller zum Entstehen neuer Ordnungen überrascht es nicht, dass die in den Mittelpunkt gestellte Epoche 1850-1890 in ihrer inhaltlichen Breite der literaturtheoretischen Diskussion nicht überbietbar erscheint. Auf allen Gebieten kann man im genannten historischen Rahmen die überkommenen und die kommenden Weltentwürfe sowie deren literaturtheoretischen Überbau gleichzeitig studieren, denn den von vielen Literaturhistorikern suggerierten radikalen Schnitt des Wendejahres 1868 hat es so nicht gegeben. ${ }^{24}$ Im Falle der Literaturtheorie erleben wir im Betrachtungszeitraum vielmehr gleichzeitig ein unversöhnliches Aufeinanderprallen wie auch die Synthese traditioneller und moderner Thesen, die einerseits auf den klassischen „Chinastudien“ (kangaku 漢学) und den klassischen „Landesstudien“ (kokugaku 国学), andererseits auf den Philosophien der europäischen Aufklärung, des deutschen Idealismus wie auch auf den Literaturtheorien der Romantik und des Realismus Europas fußen. In der Literaturgeschichte des Landes

schlechterdings nicht um den vermeintlichen Wert oder Unwert eines Textes gehen, es muss vielmehr darum gehen, was uns dieser Text über seine Zeit sagt.

21 Im Rahmen einer allgemein historischen Abhandlung wäre der Begriff „Feudalismus“ sicherlich einer tiefergehenden Erörterung zu unterziehen. Aber auch HALL 1974 und IKEGAMI 2001 (um nur zwei Beispiele zu nennen) rechtfertigen nicht, den zweifellos nur eingeschränkt auf den japanischen Fall übertragbaren Begriff „Feudalismus“ zu vermeiden.

22 JANSEN 1989.

23 HIRAKAWA 1989.

24 MAEDA 1972 (Kinsei kara kindai e 近世から近代へ [Von der frühen Neuzeit zur Frühmoderne]) kritisiert die verbreitete Behandlung der Literatur der Edo-Zeit als „Erblast“ und (soweit sie in der Meiji-Zeit in Genres und Erzähltechniken fortlebte) Modernisierungsbremse als schon deswegen haltlos, weil sie die Literaturgeschichte der späten Edo-Zeit auf die Geschichte der unterhaltenden Erzählprosa (gesaku) reduziere (3-5). MAY 1989 schildert den fließenden literaturgeschichtlichen Übergang zwischen der Edo-Zeit und der Meiji-Zeit in Form einer imaginierten „Zeitreise“ Bakins durch die Buchläden der frühen Meiji-Zeit. 
gelten die Jahre 1850-1890 als Phase der allmählichen Emanzipation der Literatur, die sich gegenüber den überkommenen Vorstellungen, im schlimmsten Falle nutzlose Unterhaltung, im besten Falle moralische Unterstützung der staatsbürgerlichen Erziehung zu sein, zu einer eigenständigen, die gesellschaftlichen Verhältnisse reflektierenden und beeinflussenden Kunstform profiliert. ${ }^{25}$ Die angedeutete inhaltliche Vielfalt der literaturtheoretischen Diskussion zeigt sich darin, dass beinahe gleichzeitig eine so stereotype Verteidigung fiktionaler Texte als Moralschule wie die obigen Texte und eine frühe Neubestimmung des literarischen Realismus im Tansō shiwa 淡空詩話 ([Tansōs Gespräche über die Poesie], 1856, erschienen 1883) des kanshi-Dichters Hirose Tansō 広瀬淡空 (1782-1856) veröffentlicht wurden. Ebenso wichtige Texte bilden den Abschluss des Betrachtungszeitraums, als prominenter Text das auf die Emanzipation des Romans als Kunstform angelegte Shōsetsu shinzui 小説神髄 (The Essence of the Novel, 1885/86) von Tsubouchi Shōyō 坪内逍遥 (1859-1935), als einer der progressivsten Texte die erste Annäherung an Hegels Ästhetik im Shōsetsu sōron 小説総論 (Allgemeine Abhandlung über den Roman, 1886) des Futabatei Shimei. In den Jahren 1885 bis 1890 vergrößerten sich die aus Autorschaft und Leserschaft gebildeten Kreise literaturtheoretischer Diskutanten um ein Vielfaches, unter anderem durch die rasant steigende Zahl lesefähiger Menschen, die Vermassung der literarischen Produktion sowie die Individualisierung des Leseverhaltens ${ }^{26}$ und nicht zuletzt durch die Gründung wichtiger Zeitschriften mit literaturtheoretischen Schwerpunkten. Um nur einige wenige zu nennen: In der 1885 gegründeten Zeitschrift Chūō gakujutsu zasshi 中央学術雑誌 erschienen herausragende Arbeiten von Tsubouchi Shōyō, Futabatei Shimei und Takada Hanpō 高田半峰 (1860-1938), in der ebenfalls 1885 gegründeten Jogaku zasshi 女学雑誌 wurden neben den Arbeiten von Ishibashi Ningetsu 石橋忍月 (1865-1926) solche von Uchida Roan 内田魯庵 (1868-1929) und Kitamura Tōkoku 北村透谷 (1868-1894) veröffentlicht, die 1887 gegründete Zeitschrift Kokumin no tomo 国民之友 präsentierte neben zahlreichen allgemein politischen Artikeln auch wichtige literaturtheoretische Arbeiten von Tokutomi Sohō 徳富蘇峰 (1863-1957), Tokutomi Roka 徳富蘆花 (1868-1927), Mori Ōgai 森鴎外 (1862-1922) und Uchida Roan. 1889 erschien mit der von Mori Ōgai gegründeten Shigarami zōshi しがらみ草紙 die erste auf Literaturtheorie und -kritik spezialisierte Zeitschrift. Die Herausbildung und Festigung der japanischen Literaturtheorie erfährt hierdurch einen ersten Höhepunkt, der es rechtfertigt, den Betrachtungszeitraum hier zu beschließen.

25 Über den Emanzipationsprozess informiert ausführlich SCHAMONI 1975.

26 MAEDA 1973 [1962] und MAEDA 1973. 


\subsection{Das zeitgenössische Textsortensystem der japanischen Literatur, die Terminologie, das Kernkorpus und die Anlage der Abhandlung}

\subsubsection{Das zeitgenössische Textsortensystem der japanischen Literatur}

Wer im literaturhistorischen Vorfeld der Betrachtungszeit 1850-1890 vom 10. Jahrhundert bis zum 19. Jahrhundert unvoreingenommen und ohne die Einschränkung auf die Dichotomie „Fiktion - Wirklichkeit“ über alle Textsorten hinweg nach japanischen Literaturtheorien sucht, wird nicht nur durch zahllose wertvolle Funde belohnt - das Feld erschiene geradezu unendlich weit und unüberschaubar, böte nicht die in Kapitel 2 besprochene Forschungsliteratur die unabdingbare erste Orientierung. Die unendlich scheinende Weite des Feldes ,japanische Literaturtheorie“ ergibt sich daraus, dass schaffensästhetische, werkästhetische und rezeptionsästhetische Literaturtheorien in Texten der Ethik und der Moralphilosophie zu finden sind, in Texten zur Reform des Bildungswesens, in Erlassen zum Verlagswesen sowie in Begleittexten (Widmungen, Motti, Leitsätzen, Vor- und Nachworten) zu literarischen Texten. Literaturtheorien sind Teile unterschiedlichster Bestrebungen, die japanische Gesellschaft in ihrer Gesamtheit zu stabilisieren oder zu reformieren, und schließlich üben sie auch Einfluss auf Produktion und Rezeption der Kunst aus. Eine derartige Breite möglicher Zielsetzungen verleiht der Literaturtheorie an sich großes gesellschaftliches Gewicht.

Dieses gesellschaftliche Gewicht wird noch dadurch gesteigert, dass in benachbarten Teilfeldern der Kunst ähnliche Anschauungen wie bei der Literatur (im engeren Sinne) ihren theoretischen Niederschlag finden. Wenigstens ein Beispiel aus dem Bereich der bildenden Kunst soll dies verdeutlichen. Der neokonfuzianische Gelehrte Asaka Gonsai 安積艮斎 (1791-1861) schuf auf Wunsch seines Gelehrtenkollegen Ōtsuka Soken 大塚素軒 (1821-1874) den „Leitspruch zum Gemälde eines Tigers, dem Wunsche des Ōtsuka Soken entsprechend“. ${ }^{27}$ Darin lobt er, dass der Künstler sich nicht auf die Darstellung des Äußeren des Tigers konzentriert, sondern das Wesen verdichtet (shin wo korasu 凝神) und seine eigenen Neigungen zurückgenommen (kokoro wo hisomu 潜心) habe. Die Hymne auf das Kunstwerk gipfelt in der Festellung, der Künstler habe seine wunderbare Gestaltung gemeinsam mit dem Schöpfer und Wandler ersonnen. ${ }^{28}$

27 Originaltitel: Gako no zu ni dai shite Ōtsuka Soken no motome ni ōzu 題画虎図応大塚素軒之索. ASAKA-KIKUTA-ANDŌ 2010 [1853]: 59-62.

28 „Schöpfer und Wandler“ für zōka 造化 (chin. zaohua). Diese Formulierung und die Variante zōbutsusha 造物者 (chin. zaowu zhe, „Schöpfer der Dinge“) sind im Gonsai shiryaku (der Sammlung, in der auch der hier erwähnte Text enthalten ist) häufig anzutreffen und gehen 
Gonsai bezieht sich damit für seine „Kunsttheorie“ wie viele der später in dieser Abhandlung zu besprechenden Literaturtheoretiker auf den selben kanonischen Text der daoistischen Philosophie Chinas: das Zhuangzi 荘子 (The complete works of Chuang-tzu) des Zhuang Zhou 荘周 (365-290 v. Chr.). Gonsais (argumentativ allerdings schwache) „Kunsttheorie“ ist in der Mitte des 19. Jahrhunderts kein Einzelfall mehr, wie die hier aus Platzgründen nicht näher ausführbaren Seitenblicke in das Nachbarfeld erwiesen haben. Die Seitenblicke vermittelten den Eindruck, dass das Feld „Kunst“ begonnen hat, sich zu einem autonomen Teilfeld der japanischen Kultur zu entwickeln. Die in dieses Teilfeld eingeschlossenen Bereiche der bildendenden und der sprachlichen Kunst gründen nun allmählich auf einer übergreifenden (und natürlich nicht aus dem Zhuangzi allein bestehenden) philosophischen Basis ihren theoretischen Überbau und beanspruchen in diesem Übergreifen gesellschaftliches Gewicht.

Zur oben angedeuteten gesellschaftlich bedingten Breite des Feldes „Literatur" kommt aber noch dessen Diffusion in den verfügbaren Metainformationen hinzu, denn japanische Literaturtheorien werden auch in modernen Recherchemitteln häufig mit Literaturkritik und allgemeiner Kritik (bungei hihyō 文芸批評, hihyō 批評) auf eine Stufe gestellt ${ }^{29}$ und nicht von jenen Textgruppen getrennt gehalten. In Standardwerken zur japanischen Literaturgeschichte sowie in den daraus exzerpierten chronologischen Tabellen verstecken sich in der Tat veritable Literaturtheorien häufig in der Sparte hyōron 評論 („Wertung und

zurück auf das oben erwähnte Zhuangzi. Als locus classicus gilt der Abschnitt Da zongshi 大宗 師 („Der große Ahn und Meister“) im ersten Buch (Neibian 内編, „Esoterisches“). Dort unterhalten sich vier dem Tode nahe Freunde darüber, was der „Schöpfer der Dinge“ aus ihren Körpern oder Teilen davon nach dem Tode als nächstes schaffen wird. Das Loblied auf die Kreativität des Schöpfers gipfelt in dem Diktum: 今一以天地為大鑪。以造化為大治。悪乎往 而不可哉。(Zhuangzi zhu 莊子注 3: 12b, SKQS-Edition). „Nun ist die Natur der große Schmelzofen, der Schöpfer ist der große Gießer: wohin er mich schickt, soll es mir recht sein“ (ZHUANGWiLHELm 1992 [1912]; [2. Jh. v. Chr.]: 51). Diese chinesische Vorstellung eines Schöpfers, der aus dem in der Natur vorhandenen Material Neues schafft, findet sich in der japanischen Philosophie schon seit dem 13. Jahrhundert (etwa im Shōbō genzō des Dōgen, 1231). Zwar wird zōka in der Kunst und der Literatur Japans, beispielsweise in den Reisetagebüchern des Matsuo Bashō (17. Jh.), häufig als Synonym für shizen 自然 („das aus sich selbst so Seiende“, Natur), also das Grundmaterial neuer Schöpfungen selbst, verwendet; hier aber steht es für die Schöpfergottheit, mit welcher der Künstler als zweiter Schöpfer verglichen wird. Von einer bloßen Abbildung der Natur ist dadurch der Schöpfungsakt des Künstlers deutlich unterscheidbar.

29 Japan steht innerhalb Ostasiens mit dem Übergehen von Literaturtheorie in Literaturkritik nicht allein. OWEN 1992 und POHL 2007 konstatieren in etwa die gleichen Erscheinungen in China, auf die ich im Verlauf dieser Abhandlung noch zurückkommen werde. HIJIYA-KIRSCHNEREIT 1979 attestiert der modernen japanischen Literaturgeschichtsschreibung „Theoriedefizit und Wertungswut“. 
Diskussion“) - auf derselben Ebene wie Texte, die Impressionen ohne jeglichen argumentativen Überbau enthalten. ${ }^{30}$ Das gilt im Prinzip auch für historische Standardwerke der Literatur und der Literaturtheorie im Allgemeinen sowie der Buch- und Verlagsgeschichte im Besonderen, für Textsammlungen, Fachlexika, chronologische Tabellen, Bestandsverzeichnisse privater und öffentlicher Bibliotheken, zeitgenössische Publikationsverzeichnisse ${ }^{31}$ sowie für Volltextdatenbanken. ${ }^{32}$ Zwar entwirft nun die Forschungsliteratur speziell zu den hyōron ein differenzierteres Bild als chronologische Tabellen es könnten. Doch auch die Standardwerke zur Geschichte der Literatur und der hyōron erweisen sich mitunter als zu unspezifisch oder umgekehrt als Prokrustesbett, weil sie beispielsweise traditionelle Kanones nicht in Frage stellen, geschweige denn erweitern. ${ }^{33}$ Man fürchtet also wohl zu Recht, nur ein diffuses und unspezifisches Bild von der Literaturtheorie Japans zu erhalten oder gar dem falschen Eindruck zu erliegen, dass es eine solche überhaupt nicht gebe - so lange wir uns ganz auf die Metainformationen verlassen. Es wird sich zeigen, dass weder der Umfang eines Textes noch seine soziale oder textsystematische Verortung noch Form und Ästhetik das jeweils alleinige aus- oder einschließende Kriterium für japanische Literaturtheorie sein können - und schon gar nicht die Einordnung eines Textes in die Sparte hyōron oder der Ausschluss eines Textes aus derselben durch die japanische Forschungsliteratur. So kommt man schließlich dazu, auf die literaturbezogenen Metatexte unabhängig von den erwähnten Sekundärquellen auch selbstständig zu recherchieren und ein nach „westlicher“

30 Ichiko Teiji trennt nicht einmal die heterogene Gattung hyōron von literarischen Texten, sondern bringt sie für den Zeitraum 1847-1867 in der Zeile kanshibun sono ta 漢詩文・その他 („Texte in sinisierender Lyrik und Prosa sowie übriges“) unter (IснIко 1986: 224-233), zusammen mit Untersuchungen zur Sprache, sinisierenden Gedichten, philosophischen Arbeiten der „Landesstudien“ (kokugaku) sowie Miszellen (zuihitsu). Einige im definierten Sinne literaturtheoretische Texte finden sich in Iсніко 1986 unter den zuihitsu, andere unter kanshi, so etwa Shizandō shiwa 詩山堂詩話 ([Gespräche des Shizandō über Poesie], 1850). Hyōron aber gäbe es nach IснIко 1986 erst ab dem Jahr 1868.

31 Stellvertretend für andere sei hier das Kotenseki sōgō mokuroku 古典籍総合目録 ([Gesamtverzeichnis der klassischen Schriften], http://base1.nijl.ac.jp/ tkoten/about.html) des Kokubungaku kenkyū shiryō kan 国文学研究資料館 (National Institute of Japanese Literature) (künftig kurz „KTSM“) genannt.

32 Stellvertretend für viele andere seien hier die regelmäßig frequentierten Datenbanken der Nationalbibliotheken (Japan: http://dl.ndl.go.jp/ und http://kindai.ndl.go.jp/; Großbritannien: http://www.bl.uk/\#; Frankreich: http://gallica.bnf.fr/) sowie die Datenbanken übernationaler, privater und öffentlicher Bibliotheken und Forschungseinrichtungen (http://www.wul.wa seda.ac.jp/kotenseki/, http://crossasia.org/ und http://www.theeuropeanlibrary.org) genannt. 33 Diesem Punkt gehe ich in der Diskussion des Forschungsstandes (Kapitel 2) weiter nach. 
und japanischer Seite hin korrigiertes Bild davon zu entwerfen, was und wie japanische Literaturtheorie sei.

Schränkt man die Suche auf von Literaturkritik abgegrenzte Literaturtheorien speziell zur Dichotomie „Fiktion - Wirklichkeit“ ein, wird das Feld überschaubarer. Die Funde verdichten sich nun nicht nur zu einer systematischen Übersicht über vorherrschende inhaltliche Richtungen, sondern auch zu dem Eindruck, dass bestimmte literarische Formen von den Zeitgenossen als „besonders theoriewürdig“, andere als „,beschränkt theoriewürdig“ und wieder andere als ,überhaupt nicht theoriewürdig“ betrachtet worden sein müssen. Der Versuch einer Erklärung dieser Beobachtung führt zum Problem der Textsortenlandschaft in der japanischen Literatur. Wegweisend sind hier YANAGIDA 1965, SCHAMONI 1992 und SCHAMONI 2000 mit ihren Thesen zum sozialen Prestige literarischer Genres in den Jahren 1850 bis $1890 .{ }^{34}$ Für das Jahr 1850 weisen Schamonis grafische Darstellungen den Bereichen der Lyrik und Prosa in chinesischem und klassisch-japanischem Stil ein hohes soziales Prestige zu, der fiktionalen Prosa und der Theaterliteratur ein niedriges. Bungaku umschließt bei Schamoni Poesie und Prosa in chinesischem Stil sowie die konfuzianischen Klassiker der Felder Historie, Philosophie, Politik und Philologie. ${ }^{35}$ SCHAMONI 1992 und 2000 entsprechen damit dem von YANAGIDA 1965 entwickelten „Oben - Unten“-Modell (siehe unten Abschn. 2.4).

Nun durfte zwar für die Zusammenstellung eines Korpus aus literaturtheoretischen Texten kein Feld der Textlandschaft von vorneherein ausgeschlossen werden, doch fällt auf, dass sich auch nach breitestmöglicher Suche die Metatexte des Korpus wenigstens für den Zeitraum 1850-1868 weit überwiegend auf Lyrik, zu einem kleineren Teil auf Prosa und kaum auf Dramatik beziehen. Daneben kamen aber auch Texte in Frage, welche keinerlei Eingrenzung auf eine der ge-

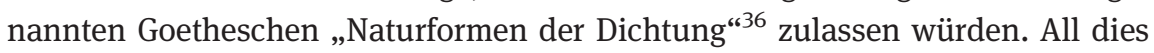

34 Schamoni 2000: 54-55. Schamonis Darlegung verwendet die Vorstellung von dem jeweils verschiedenen „Sitz im Leben“ einzelner Textsorten. (SCHAMONI 2000: 40-41; Anm. 6). Dieser vom Theologen Hermann Gunkel (1862-1932) geprägte Ausdruck bezieht sich auf die soziale Trägerschaft der Texte (WAGNER 1996) und korrespondiert darin mit dem von Yanagida Izumi entwickelten Konzept von der gesellschaftlich „oberen Literatur“ (jō no bungaku 上の文学) und der gesellschaftlich „unteren Literatur“ (ge no bungaku 下の文学). (YANAGIDA 1965 I: 34-37).

35 SCHAMONI 2000: 54.

36 In Abschnitt [318] in Noten und Abhandlungen zu besserem Verständnis des West-östlichen Divans (1819) versäumt Goethe allerdings nicht, Überlappungsmöglichkeiten zu erwähnen: „Es gibt nur drei echte Naturformen der Poesie: die klar erzählende, die enthusiastisch aufgeregte und die persönlich handelnde: Epos, Lyrik und Drama. Diese drei Dichtweisen können zusammen oder abgesondert wirken“ (GoETHE-Trunz 1998-II: 187; Sperrdruck im Original). 
legt mit Blick auf YANAGIDA 1965 sowie Schamoni 1992 und Schamoni 2000 den Schluss nahe, dass „Theoriewürdigkeit“ für die Zeitgenossen mit dem sozialen Prestige der literaturtheoretisch begleiteten Haupttexte eng verbunden gewesen sein muss. In der Tat bestätigt das Korpus dieser Abhandlung diesen Eindruck, denn für Texte mit niedrigem sozialen Prestige, namentlich kyōka (,verrückte Lieder“, eine humoristische Spielart des Waka), kyōbun (,verrückte Texte“, Begleittexte zu kyōka), gōkan (,zusammengebundene Bildhefte“) sowie die zur oralen Literatur gehörenden Genres kōdan (,vorgetragene Geschichten“) und rakugo („fallengelassene Wörter“, wortspielreiche, mündliche Erzählungen) waren im Betrachtungszeitraum keine nennenswerten Literaturtheorien zur Dichotomie „Fiktion - Wirklichkeit“ auszumachen. Dem Grund dafür, dass Theoretiker der Jahre 1850 bis 1867 diese Textsorten womöglich für „theorieunwürdig“ hielten, kann man sich nur in riskanten Spekulationen nähern, da - soweit zu sehen seitens der Theoretiker keine expliziten Erklärungen dazu vorliegen. Kyōka und kyōbun hat man vielleicht ausgeschlossen, weil grundsätzliche Fragestellungen in der unter Waka subsummierten Lyrik ohnehin seit 1000 Jahren diskutiert wurden. Gōkan wurden womöglich wegen des weit überwiegenden Bildanteils aus der Diskussion der Wortkunst ausgeschlossen. Die rakugo wurden vielleicht aus der literaturtheoretischen Diskussion der Dichotomie „Fiktion - Wirklichkeit“ ausgeschlossen, weil bei ihrer Diskussion traditionell das Hauptgewicht auf der performativen Fähigkeit des Vortragenden gesehen wurde. Auch fällt auf, dass für den Zeitraum 1850 bis 1867 keine im engeren Sinne literaturtheorische Behandlung der Dichtomie „Fiktion - Wirklichkeit“ in Bezug auf die japanische Dramatik (Nō, Jōruri, Kabuki) ausgemacht werden konnte. Hier gilt im Prinzip dasselbe wie bei den oben erwähnten rakugo: Man kann nur annehmen, dass der Schwerpunkt einschlägiger Metatexte auf der performativen Fähigkeit der Ausführenden lag. Im ganzen scheint NISHIYAMA 1972 diese Annahme zu plausibilisieren, ${ }^{37}$ so dass Texte wie die auf das Jōruri-Theater bezogene Vorrede (hottan 発端) des Naniwa miyage (1773) von Miki Heiemon Sadanari 三木平右衛門貞成

37 NishIYAMA 1972 legt in seiner Abhandlung dar, dass neben den Metatexten zu Kunsthandwerk und Akrobatik auch die Metatexte zu den darstellenden Künsten der Edo-Zeit vor allem Modelle und Methoden ( $k a t a$ 型 und $h \bar{o}$ 法) des Körpereinsatzes zu vermitteln suchen. Gründe für diesen inhaltlichen Schwerpunkt sieht Nishiyama in dem Bestreben der Verfasser, die Techniken ihrer „Schule“ für die nächste Künstlergeneration im eigentlichen Sinne festzuschreiben und weiterzugeben, sowie in der Herkunft der darstellenden Künste aus der landwirtschaftlichen Produktion und der darin geprägten, aus seiner Sicht einmaligen japanischen Körperlichkeit. Als Beispiel dient etwa die nanba ナンバ (seltener 南蛮 geschrieben) genannte Fortbewegung, in welcher der linke Arm und das linke Bein parallel bewegt werden. NishIYAMA 1972 legt dar, dass Metatexte zu den darstellenden Künsten der Edo-Zeit auch dazu dienen sollten, Techniken wie nanba durch schriftliche Bewahrung gegen vielfältige ausländische Einflüsse zu bewahren. 
(Lebensdaten und biografische Details unbekannt) und Hozumi Ikan 穂積以貫 (1692-1769) als Ausnahme zu sehen wären. ${ }^{38}$

Allerdings vollzog sich ab dem Jahre 1868 in Japan eine historische Wende, nach der das Land sich den ökonomischen, politischen und sozialen Einflüssen Europas beinahe grenzenlos hingab. Dass hiernach auch bislang als „theorieunwürdig“ angesehene Textsorten allmählich an Prestige gewannen und zum Gegenstand anspruchsvoller theoretischer Darlegungen wurden - und vor allem: woher dieser allmähliche Prestigegewinn kam, wird diese Abhandlung zeigen.

\subsubsection{Das Textkorpus als Rahmen der Abhandlung}

Die Reduzierung der in Frage kommenden Menge an Literaturtheorien auf ein „Korpus“ bezweckt zuallererst, eine noch von einem einzelnen Forscher bearbeitbare Auswahl an Beispieltexten zusammenzustellen. Die dazu notwendige Auswahl musste grundsätzlich drei Bedingungen erfüllen. Vor allem mussten die Metatexte die Wortkunst („Literatur“) zum Gegenstand haben und als Literaturtheorie einzustufen sein. Zweitens mussten sie die erwähnte Vielfalt der Textsorten, der sozialen Träger und der Leserschichten berücksichtigen. Die anhand der vorgenannten Auskunftsmittel, der einschlägigen Sekundärliteratur und im Blick darüber hinaus erschlossenen Literaturtheorien waren miteinander zu vergleichen, bis strukturelle und inhaltliche Paradigmen erahnt und anhand weiterer in einem heuristischen Verfahren ausgewählter Texte bestätigt, korrigiert oder ergänzt werden konnten.

Das Korpus derjenigen Texte, welche musterbildend für den Betrachtungszeitraum gewesen sein können, verzeichne ich im Original und in Übersetzung unter der Bezeichnung „Primärquellen“ (Abschn. 6.5) im Literaturverzeichnis, das Korpus der im Mittelpunkt der vorliegenden Abhandlung stehenden Literaturtheorien nenne ich „Korpus“ (Abschn. 6.4). Prominente Texte wie die Erörterung des Fiktionalen im Kapitel Hotaru des Genji monogatari (Die Geschichte vom Prinzen Genji, ca. 1010) ${ }^{39}$ konnten nicht in die Gruppe „Primärquellen“ aufgenommen werden, denn dieser und viele ähnliche Texte propagieren entweder eine Substitution moralischer Begriffe, etwa die Substitution von „Verlogenheit“ (uso) und „Wahrhaftigkeit“ (makoto), oder sie bleiben in deren Abgrenzung gegen nicht moralische Begriffe zu diffus (QUENZER 2008). Texte dieser Kategorie definieren aber nicht den im weitesten Sinne semasiologischen Zusammenhang zwi-

38 REGELSBERGER 2004.

39 UEDA 1991: 25-36. 
schen „Fiktion“ und „Wirklichkeit“ und unterscheiden sich dadurch von Texten wie dem noch zu besprechenden Hōbutsu shū (1179).

Im Verlauf der Recherche entstand aus der Gruppe von 450 nach obigen Bedingungen ausgewählten Kandidaten ein 88 Texte umfassendes Korpus. Bei der Auswahl der Korpustexte orientierte ich mich für den Zeitraum der späten EdoZeit vor allem an der erwähnten „Oben - Unten“-Struktur aus YANAGIDA 1965-I und deren Schichtung in SCHAMONI 1992 und SCHAMONI 2000. ${ }^{40}$ Die literaturtheoretischen Überlegungen wurden dabei sowohl aus Begleittexten (Überschriften, Vor- und Nachworten) als auch aus Haupttexten (Monografien, theoretisierenden Einschüben des Autors) entnommen. ${ }^{41}$

Tabelle 3: Kandidaten und Korpus.

\begin{tabular}{llrrrrr}
\hline Phase & Jahre & Kandidaten & & \multicolumn{3}{c}{ Korpus } \\
\cline { 6 - 8 } \cline { 5 - 6 } & & Anzahl & & Anzahl & jō (Prestige hoch) & ge (Prestige niedrig) \\
\hline $\mathbf{1}$ & $1850-1867$ & 88 & & 41 & 16 & 25 \\
\hline $\mathbf{2}$ & $1868-1890$ & 362 & 47 & - & \\
\hline & Summen & 450 & & $\mathbf{8 8}$ & & \\
\hline
\end{tabular}

Phase 1 (1850-1867) ist mit 88 Kandidaten zu nicht einmal einem Viertel vertreten, im Korpus liegen jedoch die Titelzahlen der beiden Phasen näher beieinander, was der beabsichtigten Gleichgewichtung der Texte entspricht. Dies soll einen Mangel ausgleichen: Texte der späten Edo-Zeit sind wesentlich schlechter erschlossen, so dass von der Zahl der ermittelten Titel nicht verlässlich auf deren exemplarische Vielfalt geschlossen werden könnte. Viel sicherer ließ sich dagegen diese Vielfalt bei der Kandidatengruppe der Phase 2 (1868-1890) annehmen. Insgesamt schienen die systematischen Möglichkeiten der Literaturtheorie des gesamten Betrachtungszeitraums anhand des Korpus ausgeglichen erforschbar.

40 YANAGIDA 1965 gehört mit seiner minutiösen Nachzeichnung der Entwicklung des literarischen Denkens (bungaku shisō 文学思想) der frühen Meiji-Zeit (1868-ca. 1890) zu den unverzichtbaren Grundlagen meines Unternehmens. SCHAMONI 2000 erweitert Yanagidas Betrachtungszeitraum rückwärts bis ca. 1850 und beschreibt den dort beginnenden Wandel der Textsortenlandschaft. Es soll nicht unerwähnt bleiben, dass Maeda Ai die von Yanagida unternommene Schichtung nach „oben - unten“ mit der in NAKAMURA 1961 enthaltenen Schichtung nach „edel“ ( $g a$ 雅) und „vulgär“ (zoku 俗) durchaus überzeugend gleichsetzt (MAEDA 1972: 5). Ich gebe Yanagidas Schichtung gleichwohl wegen ihres wertneutralen Charakters den Vorzug.

41 Quantitative und qualitative Details des Korpus wurden im vorausgehenden Abschnitt (1.3.1, S. 16 ff.) beschrieben. 
In der vorliegenden Abhandlung werden nicht alle einzelnen Texte, sondern nur 14 Beispiele des Korpus direkt miteinander kontrastiert.

\subsubsection{Terminologie: die einfache Dichotomie „Fiktion - Wirklichkeit“ und die Iser'sche Triade „Fiktion - Wirklichkeit - das Imaginäre“}

Die in ISER 1993 eingeführte Triade „Fiktion - Wirklichkeit - das Imaginäre“ erscheint als terminologisches System auf den ersten Blick methodisch attraktiv und soll deswegen durch das folgende Zitat so kurz als möglich dargestellt werden.

Das Oppositionsverhältnis von Fiktion und Wirklichkeit würde die Diskussion des Fiktiven im Text um seine entscheidende Dimension verkürzen; denn offensichtlich gibt es im fiktionalen Text sehr viel Realität, die nicht nur eine solche identifizierbarer sozialer Wirklichkeit sein muß, sondern ebenso eine solche der Gefühle und Empfindungen sein kann. Diese gewiß unterschiedlichen Realitäten sind ihrerseits keine Fiktionen, und sie werden auch nicht zu solchen, nur weil sie in die Darstellung fiktionaler Texte eingehen. Andererseits ist es jedoch ebenso zutreffend, daß solche Wirklichkeiten, wenn sie im fiktionalen Text auftauchen, dort nicht um ihrer selbst willen wiederholt werden. Bezieht sich also der fiktionale Text auf Wirklichkeit, ohne sich in deren Bezeichnung zu erschöpfen, so ist die Wiederholung ein Akt des Fingierens, durch den Zwecke zum Vorschein kommen, die der wiederholten Wirklichkeit nicht eignen. Ist Fingieren aus der wiederholten Wirklichkeit nicht ableitbar, dann bringt sich in ihm ein Imaginäres zur Geltung, das mit der im Text wiederkehrenden Realität zusammengeschlossen wird. So gewinnt der Akt des Fingierens seine Eigentümlichkeit dadurch, daß er die Wiederkehr lebensweltlicher Realität im Text bewirkt und gerade in solcher Wiederholung das Imaginäre in eine Gestalt zieht, wodurch sich die wiederkehrende Realität zum Zeichen und das Imaginäre zur Vorstellbarkeit des dadurch Bezeichneten aufheben. Daraus läßt sich folgern, daß die triadische Beziehung des Realen, Fiktiven und Imaginären eine basale Beschaffenheit des fiktionalen Textes verkörpert. Gleichzeitig wird deutlich, was den Akt des Fingierens und damit das Fiktive des fiktionalen Textes auszeichnet. Wird die im Fingieren wiederholte Realität zum Zeichen, dann geschieht zwangsläufig ein Überschreiten ihrer Bestimmtheit. Der Akt des Fingierens ist folglich ein solcher der Grenzüberschreitung. ${ }^{42}$

Attraktiv ist die Triade als terminologisches Instrument durch die Unterscheidung zwischen extraliterarischer (sozialer und emotionaler) und intraliterarischer Wirklichkeit, wobei letztere eine verfremdete Auswahl aus ersterer darstellt. Die Differenz extraliterarischer und intraliterarischer Wirklichkeit stellte sich damit als „das Imaginäre“ dar und lieferte dann den Zugang zur Intention des Verfassers eines literarischen Textes.

42 ISER 1993: 19-21. 
Nun ließe sich das triadische Modell auf die Primärtexte der japanischen Literatur zweifellos gewinnbringend anwenden. Beispielsweise könnten wir die von Ihara Saikaku 井原西鶴 (1642-1693) in seinen Romanen des Genres ukiyo$z \bar{s}$ shi wiederholte Wirklichkeit des bürgerlichen Lebens mit der in zeitgenössische und moderne extraliterarische Historiografien eingeflossenen Wirklichkeit des bürgerlichen Lebens abgleichen. Das würde zumindest dann zu einer spezifischen Differenz führen, wenn man die in zeitgenössische und moderne Historiografien eingeflossene Wirklichkeit als wenigstens annähernd vollständig wie unverzerrt, und eben nicht als „literarisches Kunstwerk“ betrachten dürfte. Vor dem Hintergrund der in WHITE-KOSELLEK 1991 dargelegten Überlappung zwischen Historie und Narration („Auch Klio dichtet“) ist der Erwartung einer spezifischen Differenz zwischen intra- und extraliterarischer Wirklichkeit freilich mit Skepsis zu begegnen. ${ }^{43}$

Auf die Sekundärtexte der japanischen Literatur, im engeren Sinne auf die Literaturtheorien, ließe sich hingegen die Iser'sche Triade „Fiktion - Wirklichkeit - das Imaginäre“ nur dann anwenden, wenn in den japanischen Theorien eine Entsprechung zum Iser’schen „Imaginären“ auszumachen wäre. Die Annahme einer dritten Dimension ist, soweit zu sehen, mangels direkter Verbalisierung und beim grundsätzlich dichotomischen Charakter einschlägiger Theorien zu spekulativ. Für unser Unternehmen müssen wir deswegen von der Anwendung des triadischen Modells absehen und uns auf die Betrachtung der Dichotomie „Fiktion - Wirklichkeit“ beschränken. ${ }^{44}$

\subsubsection{Anlage der Abhandlung}

Die vorliegende Abhandlung betritt inhaltlich und methodisch zwar Neuland, aber die bisherige Forschung stellt dafür reichlich Material und Werkzeug zur Verfügung. In Kapitel 2 (Forschungsstand und Quellenlage) diskutiere ich den Stand dieser Forschung zum umrissenen Problem sowie zur Quellenlage.

43 Vgl. insbesondere das Kapitel „Der historische Text als literarisches Kunstwerk“ (WHITEKOSELLEK 1991: 101-122).

44 Übrigens ist Isers Theorie des Imaginären keineswegs unumstritten. STRÖKER 1983 etwa bringt (wenn auch in Form rhetorischer Fragen) das „Imaginäre“ Isers in die Nähe eines theoretischen Konstrukts, das nicht aus den Gegebenheiten des fiktionalen Texts hergeleitet werden kann, sondern im aristotelischen Sinne „Materie“ als Korrelat zur „Form“ wäre. Isers Entgegnung auf STRÖKER 1983 räumt denn schließlich zwar ein, dass das Imaginäre sich gegen seine „begriffliche Erfaßbarkeit“ sperre, verteidigt das „Imaginäre“ aber doch in der These, dass Fiktives und Imaginäres zwar ständig ineinander übergingen, dabei aber nicht ihre Konturen löschten (ISER 1983: 486). 
Keine kulturelle Epoche schafft etwas Neues, ohne auf in vorausgehenden Epochen Geschaffenes zurückzugreifen oder sich darauf $\mathrm{zu}$ beziehen. Kapitel 3 (,Fiktion“ und „Wirklichkeit“: Beziehungsmuster in Philosophie und Literaturtheorie) stellt die in Philosophie und Literaturtheorie im Japan vor 1850 realisierten Beziehungen zwischen „Fiktion“ und „Wirklichkeit“ zusammen, die den Theoretikern der Jahre 1850 bis 1890 als Modelle haben dienen können. Es ist nicht immer möglich, den Rückgriff der im Mittelpunkt der Abhandlung stehenden Theorien auf diesen Modellvorrat nachzuweisen, aber wo dies möglich ist, wurde er plausibilisiert.

Mit Kapitel 4 (Fiktion und Wirklichkeit in ausgewählten japanischen Literaturtheorien der Jahre 1850 bis 1890) beginnt der Hauptteil der vorliegenden Abhandlung. In größtmöglicher exemplarischer Breite werden Literaturtheorien vorgestellt, die sich mit dem Verhältnis „Fiktion und Wirklichkeit“ in literarischen Sprachhandlungen befassen. $\mathrm{Zu}$ keiner Zeit besteht dabei die Annahme, dass die nach ihren formalen Strukturen gruppierten Theorien je als Teil eines in seiner Gesamtheit synchron funktionierenden Systems gedacht waren.

Welche Kontinuitäten und welche neue Entwicklungen in der japanischen Literaturtheorie zur Dichotomie „Fiktion - Wirklichkeit“ im Zeitraum 1850-1890 in Kapitel 4 ausgemacht werden konnten, fasst Kapitel 5 (Fazit: Von Kontinuitäten und Wandlungen in der japanischen Literaturtheorie von „Fiktion“ und „Wirklichkeit“) zusammen.

Kapitel 6 (Literaturverzeichnis) verzeichnet neben den verwendeten Hilfsmitteln auch die Korpus-Kandidaten, das Korpus, die Primärquellen (die Quellen, aus denen die Texte des Korpus geschöpft haben könnten) sowie die für die vorliegende Abhandlung verwendete Sekundärliteratur.

Kommentierte Übersetzungen (als solche unmarkierte eigene wie zitierte fremde) dienen zur Veranschaulichung der Typologie ebenso wie zur Plausibilisierung der ermittelten Paradigmen von Struktur und Inhalt der Texte. Einige der ausgewählten Texte wurden und sollen getrennt von dieser Abhandlung in kommentierter Übersetzung in Fachzeitschriften publiziert werden, einige andere Texte werden in kommentierter Übersetzung $\mathrm{zu}$ einem Teil der Abhandlung selbst. 